\title{
Integration Application of 8K Ultra HD Video and DMS Audio Broadcast System
}

\author{
Jinbao Song ${ }^{1, a}$, Chong Guo ${ }^{1}$, Xin $\mathrm{Xin}^{2}$ and Xiaoguang $\mathrm{Wu}^{2}$ \\ ${ }^{1}$ Communication University of China, Faculty of Science and Technology, School of Information Engineering, Beijing, P.R. China \\ ${ }^{2}$ Beijing HeXinChenGuang Information Technology Co., Ltd, Beijing, P.R. China
}

\begin{abstract}
In recent years, people's demand for audio and video playback quality has gradually increased. High definition video and high reduction audio are two important index. The $8 \mathrm{~K}$ ultra-high-definition television broadcasting system was developed by Beijing HeXinChenGuang Information Technology Co., Ltd. It can broadcast ultra-high-definition uncompressed video signals with a bandwidth of $8 \mathrm{~GB} / \mathrm{s}$ and a resolution of $7680 \times 4320$. The Dynamic Matrix Sound (DMS) is a new multi-channel pickup system based on the principle of sound field optimization and the Huygens principle. DMS is developed by the Key Laboratory of Media Audio Education of the Communication University of China. This paper proposes a communication method that integrates the above two systems. It mainly describes an application layer protocol based on User Datagram Protocol (UDP). It focuses on the message distribution and message transmission mechanism of the protocol. The operating conditions can achieve synchronous playback of 8K ultra-high-definition video and DMS audio. The integration application of 8K ultra HD video and DMS audio broadcast system is realized.
\end{abstract}

\section{Introduction}

Images are an indispensable part of people's daily life. The early image media was mainly television. The earliest television technology was called standard definition. The physical resolution of standard-definition TV is $720 \times 576$. Nowadays, most of the civil television has achieved high definition. There are several accepted standards for high-definition television transmission, and their physical resolutions are 720p (HD Ready): 1280 $\times 720$ p, 1080i (Full HD):1920×1080i, 1080p (Full HD): $1920 \times 1080 \mathrm{p}$. Ultra HD includes $4 \mathrm{~K}$ and $8 \mathrm{~K}$ standards. The physical resolution of $4 \mathrm{~K}$ is $3840 \times 2160$. The physical resolution of $8 \mathrm{~K}$ is $7680 \times 4320$. $8 \mathrm{~K}$ is at the forefront direction of current video technology research and the latest standard. The physical resolution of $8 \mathrm{~K}$ technology is 16 times of traditional 1080 p, four times of $4 \mathrm{~K}$. High resolution, not only brings striking visual effect and breathtaking experience, but also gives profound challenges to all aspects of $8 \mathrm{~K}$ media such as production, storage, transmission and broadcast.

Sound is an essential part when people watch video. If audio is recorded over multiple channels, we call it stereo. Early dual-channel stereo used two input channels for recording. When it was played, the output of the two channels was recorded with a sound level difference to simulate the spatial sense of sound [1]. If more than two channels are used for recording, it is called multi-channel stereo, and typically there are three channels, four channels, 5.1 channels, 7.1 channels and 9.1 channels. In

\footnotetext{
a Corresponding author: songjinbao@cuc.edu.cn
}

addition, many audio systems of more channels also appear. For example, Japan's NHK Broadcasting Technology Institute has developed audio system of 22.2 channels, compared with the traditional 5.1 channels, it can bring more shock to the listener's viewing experience [2].

The development trend of audio and video until now is $8 \mathrm{~K}$ and multichannel. An audio and video broadcast system combining $8 \mathrm{~K}$ images and multichannel audio, technically, is the most advanced technology in line with the technological trend of image development. In terms of the market, with the economic development year by year, people's consumption capacity of high-end images will increase year by year, which can meet the market demand. Therefore, this paper proposes an 8K ultra HD video and DMS audio broadcast system, which is in line with the advanced technology and the leading market, and has a strong practical significance.

\section{Related work}

In recent years, super high definition video has been widely used in many applications. NHK realized realtime transmission of $4 \mathrm{~K} / 12 \mathrm{Bit}$ image transcoding to $2 \mathrm{~K} / 8 \mathrm{Bit}$ [3]. SONY has announced that the 2020 Tokyo Olympics will be produced and broadcast using the $8 \mathrm{~K}$ standard [4]. In December 2017, BOE announced the mass production of its 10.5 th generation pipeline, marking the official production of $8 \mathrm{~K}$ display screen [5].

As a 3D playback format, 22.2 channel format is 
closer to the actual sound field than the 5.1 channel format adopted by current digital broadcasting [6]. There are solutions for increasing the number of playback devices in the commercial reproducing system, primarily such as Dolby and DTS (Dedicated to Sound).

The integration application of $8 \mathrm{~K}$ ultra HD video and DMS audio broadcast system is researched in this paper.

\section{Overview of $8 k$ UHD video and DMS audio broadcast system}

\subsection{K ultra HD TV broadcast system}

The $8 \mathrm{~K}$ ultra-high-definition television broadcasting system is developed by Beijing HeXinChenGuang Information Technology Co., Ltd. $8 \mathrm{~K}$ no compressed video player (HXH404H - S8P) is based on the ITU new standards the ITU - R BT. 2020-2 (10/2015) and ITU - R BT. 2100-0 (07/2016). 8K- HDR player can play uncompressed ultra-high video signal of $7680 \times 4320$ resolution, can play video signals of 60 frames per second to meet the requirement, can choose to play $\mathrm{YCbCr} 422$ video signal of 10/12 bit colour depth or RGB444 video signal of 8 bit colour depth, can choose to play video signal accord with BT. 2020 colour gamut standard or BT. 709 colour gamut standard, and can choose to play HDR video signal accord with PQ standard or HLG standard. Its specifications are as follows:

1. Output interface: $4 \times$ HDMI2.0;

2. Output format:

- Resolution: $7680 \times 4320$;

- Frame rate: 60P;

- Encoding format: YUV422 10/12bit (HDR), RGB444 8bit (SDR);

- Colour gamut: BT.2020、BT.709;

- HDR support: PQ, HLG, user-defined;

3. Play time: less than or equal to 16 minutes (standard configuration), which can be repeated;

4. Machine case size: $46 \mathrm{~cm}$ (long) $\times 44 \mathrm{~cm}$ (wide) $\times$ $20 \mathrm{~cm}$ (high), special machine case; $63 \mathrm{~Hz}$

5. Power source: AC100-240V (wide), 13A-6.5A, 47-

6. Working environment: $5 \sim 55^{\circ} \mathrm{C}$, relative humidity $30 \% \sim 80 \%$ (not condensation);

7. Saved environment: $-20 \sim 60{ }^{\circ} \mathrm{C}$, relative humidity $30 \% \sim 90 \%$ (not condensation).

\subsection{Dynamic Matrix Sound (DMS) system}

The Dynamic Matrix Sound (DMS) is a new multichannel pickup system based on the principle of sound field optimization and the Huygens principle. DMS is developed by the Key Laboratory of Media Audio Education of the Communication University of China from December 2015 to May 2017.

The Dynamic Matrix Sound (DMS) system uses microphone array and adaptive filter to separate different sound source signals, and adopts improved particle filter for non-Gaussian system. By separating the main finite sound sources, the analysis and representation of other sound fields are more simple and feasible. A multisolution system of $\mathrm{M}$ inputs and $\mathrm{N}$ outputs is formed by the combination of finite sound source and infinite sound field. It is a system suitable for a variety of actual environment, which is superior and more flexible than a single sound field comprehensive method. So the invention of the dynamic matrix (DMS) can realize the dynamic combination and dynamic broadcast of audio source, can create different sound and music form according to different playback scenarios and the art creation needs. Eventually, with new sound field optimization principle and Huygens principle, the system suitable for a variety of actual environment is gotten, which is superior and more flexible than the comprehensive method of traditional acoustic field.

The DMS dynamic sound system consists of the following modules: (1) recording module; (2) audio processing module; (3) hardware module of DMS system; (4) the DMS system software module; (5) broadcast module; (6) subjective evaluation of DMS system.

The key technology of DMS dynamic sound system includes: (1) sound source localization and separation; (2) data storage; (3) use speaker array to realize sound field synthesis; (4) adaptive matching technology from $M$ sound sources to $\mathrm{N}$ sound channels.

\subsection{Related technology of 8K ultra HD video and DMS audio broadcast system integration}

At the beginning of the research on multi-channel dynamic sound system (DMS), considering the performance of the platform and possible future demand of cross-platform transplantation, Qt framework development is used. Qt framework is a widely used GUI framework. It is firstly a commercial closed source framework. In 2009, Qt is announced open source after Nokia acquisition of the company. Open source makes Qt enrich and expand the basis code. The graphics interface program can be implemented smoothly, highperformance by using Qt. Qt has the following advantages:

1. Cross-platform deployment. The same set of Qt code can be compiled to binary files for different operating systems without the need to change, can run on the various hardware from the embedded devices to mainframe. These platforms include: (1)Linux/Unix: X11, Wayland, Embedded Linux, Android; (2)Apple: macOS, iOS; (3)Microsoft: Windows, Windows Phone, Windows RT, Windows CE; (4)Other embedded systems: Integrity, QNX, VxWorks; (5)Other unofficial support platforms: OpenSolaris, Haiku, OS/2, webOS, Amazon Kindle DX, Ubuntu, AmigaOS, Tizen, Sailfish OS.

2. Rich application program interface. The basic $\mathrm{c}+$ +library of Qt provides a rich functionality. These libraries almost cover the operating system and the general development of all operations. These basic library in Qt 4.0 version is divided into independent modules, which mainly includes the following contents: (1) Qt Core; (2) Qt GUI; (3) Qt Network; (4) QML. 


\section{Demand analysis and design of $8 \mathrm{~K}$ ultra HD video and DMS audio broadcast system integration}

\subsection{Aggregate demand analysis}

The ultimate goal of the $8 \mathrm{~K}$ ultra HD video and DMS audio broadcast system integration is to play video and audio streams with super high code rate synchronously. Based on this requirement, the following requirements are required in the overall design of the system:

(1) The middle layer is used for communication;

(2) The reliability of communication between the two systems must be ensured simultaneously;

(3) The response time of communication between the two systems must be short enough;

\subsection{Business demand analysis}

The overall design requirements of the $8 \mathrm{~K}$ ultra $\mathrm{HD}$ video and DMS audio broadcast system integration is illustrated above. In the actual business level, the overall demands are the needs of the business services. The end user for the system are the operators of small theatres, high-end KTVs, science and education screening venues. The necessary business requirements for this system are to open the audio and video files, to play audio and video files, to suspend audio and video files and to stop the audio and video files. It is not enough to just realize these business requirements in the aspect of system design. Many business requirements depend on the same bottom calls, or need more detailed step calls. The specific requirements are as follows:

(1) Connection availability test;

(2) Successful validation of business calls;

(3) The caller's delay recall for the failed one;

(4) The called party's re-entrant design for multiple recalls;

(5) File status synchronization.

\subsection{Overall system design}

The master-slave architecture is generally adopted between the two subsystems of the $8 \mathrm{~K}$ ultra HD video and DMS audio broadcast system, which is also called the Client-Server architecture or $\mathrm{C} / \mathrm{S}$ architecture. Master-slave architecture is intended to provide a scalable architecture in which a computer or processing process on the Internet is a client or server. The server side is characterized by a passive role (slave), waiting for requests from the client, processing requests, and sending back results. The client side is characterized by an active role (master) that actively sends requests to the server and waits until a response is received. The server can be stateful or stateless. The stateless server does not retain information between any two requests, and the stateful server remembers information between requests. The scope of this information can be global or transactional. A static HTML page server is an example of a stateless server. In this project, in order to guarantee the above connection availability, the stateful client-server architecture is adopted. DMS sound system as the client, sends a state transfer request. $8 \mathrm{~K}$ high-definition video broadcast system as a server exposes the fixed interface for the client to initiate the request.

In this client-server architecture, an application layer protocol based on UDP is used as a means of connection. It has two advantages to select UDP in transport layer: (1) It has good compatibility; (2) It is highly customizable.

UDP Service is the abstract layer responsible for UDP receiving and sending packets for DMS dynamic sound system. UDP Service is responsible for manipulating the UDP communication details of the operating system. GUI is an abstraction of the graphical interface, which is responsible for manipulating state changes and interaction details of the graphical interface. Core is an abstraction of the output control of DMS dynamic sound, which is responsible for the details of DMS dynamic sound output. GUI refers to Core and UDP Service. The interaction between GUI and Core and UDP Service is directly realized through function calls. The interaction between Core and UDPService is realized through signal and slot mechanism. UDPService exposes signals, and GUI binds these signals to the corresponding slot of Core. In the whole operation of dynamic sound system, interaction begins when the user clicks on GUI elements. GUI calls UDP Service request to make the system to transfer state. When $8 \mathrm{~K}$ video server returns the correct response, UDP Service sends the corresponding signal to call the corresponding Core slot function.

\subsection{Application layer protocol design based on UDP}

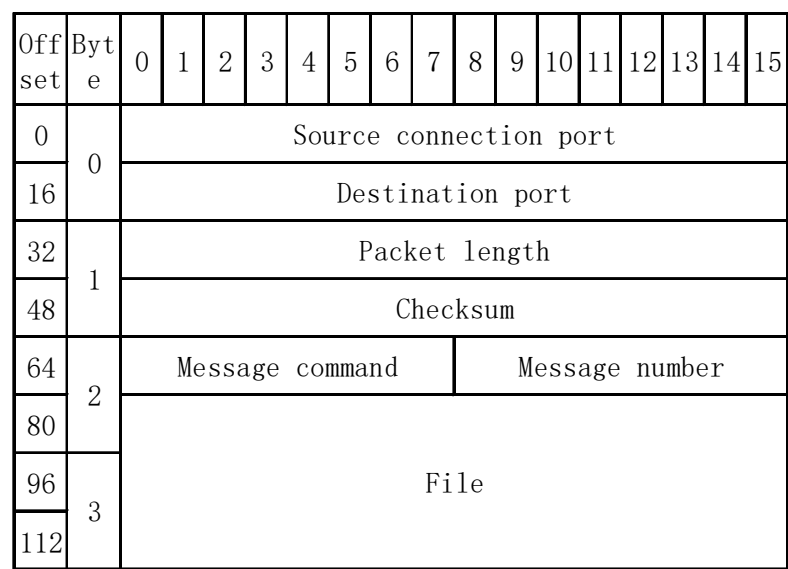

Figure 1. Application layer protocol message allocation based on UDP.

The interaction between DMS and $8 \mathrm{~K}$ ultra HD video broadcast system is realized by the application layer protocol, as shown in Figure 1. The agreement directly expands in the data section of the UDP, in data section: 0 bit to the 7 th bit are predefined message command, the eighth and 15 th bit are the message sequence number, the bits after the 16th bit are for playback file's absolute path name, its use ASCII encoding, up to 232 bit, the last eight bits are ASCII code 0x00, denoting the end of the path name. That is the file path name with variable length 
from 16 to 255 bits ends with ASCII code $0 \times 00$.

Message command is used to represent the meaning of message 8-bit unsigned integer tag, they are: handshake command 110 , open the file command 102 , play file command 104, suspend play command 106, stop playing command 108, close the file command 110 , disconnect command 112. Message response commands plus 1 in order: the response to handshake command 111 , response to open the file command 103 , response to play file command 105, response to suspend play command 107 , response to stop playing command 109 , response to close the file command 111, response to disconnect command 113.

The main demand of the $8 \mathrm{~K}$ ultra HD video and DMS audio broadcast system is to use an intermediary involved in integration without destroying encapsulation. The communication requirement is reliable and efficient. The client-server architecture is used in the design. Its communication mode uses UDP. This paper puts forward an application layer protocol based on UDP, in addition to load business requirements field, the agreement also adds some reliable transport mechanism, including two shake hands, serial number and message timeout retransmission mechanism.

\section{Implementation of $8 \mathrm{~K}$ ultra HD video and DMS audio broadcast system integration}

\subsection{The overall implementation}

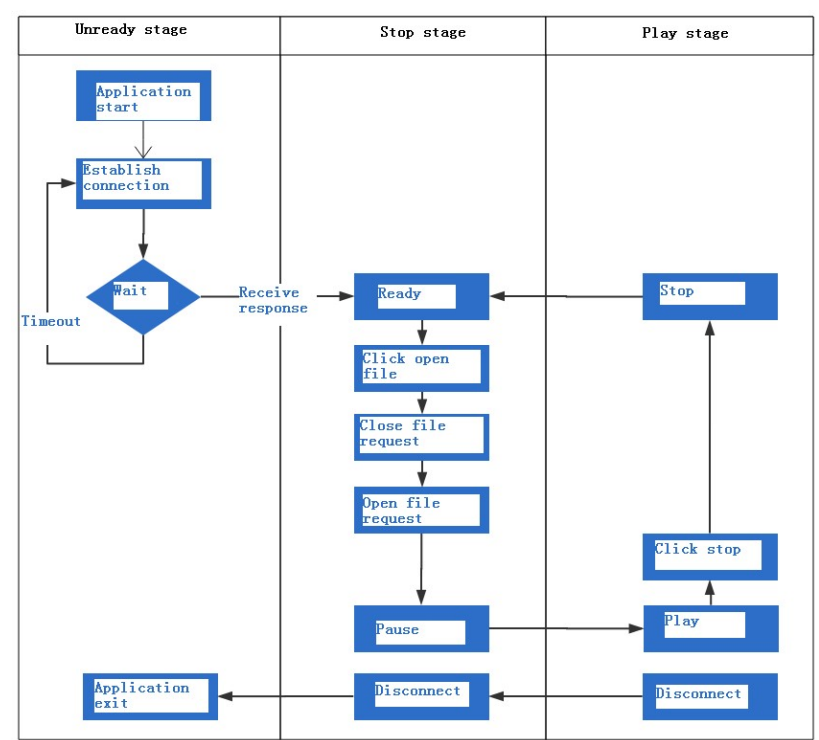

Figure 2. Flow chart of the $8 \mathrm{~K}$ ultra $\mathrm{HD}$ video and DMS audio broadcast system integration.

The overall working process of the $8 \mathrm{~K}$ ultra $\mathrm{HD}$ video and DMS audio broadcast system is shown in Figure 2. After clicking the icon, the program of DMS dynamic sound system enters the running state. BaseGUI is the main base class of the graphical interface, holding elements of the graphical interface. BaseGUI holds UDPService object and Core object, the UDPService class is responsible for UDP communication, and Core is responsible for DMS dynamic sound playback. When the three objects are initialized and related tasks have been processed, the graphical interface appears and enters the ready state. But all functionality is unavailable, because connection has not yet been established with the $8 \mathrm{~K}$ ultra HD video broadcast system at this time. After the graphical interface appears, the UDPService initiates a connection setup request. The graphical interface is continuously non-interactive while waiting for the connection to be established. Graphical interface enters into the interactive state when a connection is established. The open button can be clicked, open a file, the interface of choice is classic Windows file interface. After the file is selected, file can be played, suspended and stopped. This is the normal work process of the $8 \mathrm{~K}$ ultra HD video and DMS audio broadcast system. After work has been completed, the exit button is clicked to close the program, UDPService launches a disconnection request at this time, the program waits for a response to the normal exit. That is the normal work process of the $8 \mathrm{~K}$ ultra HD video and DMS audio broadcast system integration application.

\subsection{Implementation of UDPService}

UDPService is the core of integrated application. The main members of UDPService include two QUdpSockets, which are responsible for handling the socket details of UDP; A Qtimer, which is responsible for timeout retransmission timer; A QByteArray, which is responsible for caching the assembled message, leaving it to be read when the timeout is retransmitted to send it again; An 8-bit unsigned integer, which is responsible for recording the message sequence number.

The initialization process for UDPService takes four steps. The first step is to create the required $\mathrm{C}++$ member object. The second step is to set the message sequence number to 0 . The third step is to bind the two QUdpSocket using bool QUdpSocket::bind (const QHostAddress \& address, quint16 port) function respectively. The fourth step is to connect the signal and slot.

\subsection{Implementation of BaseGUI}

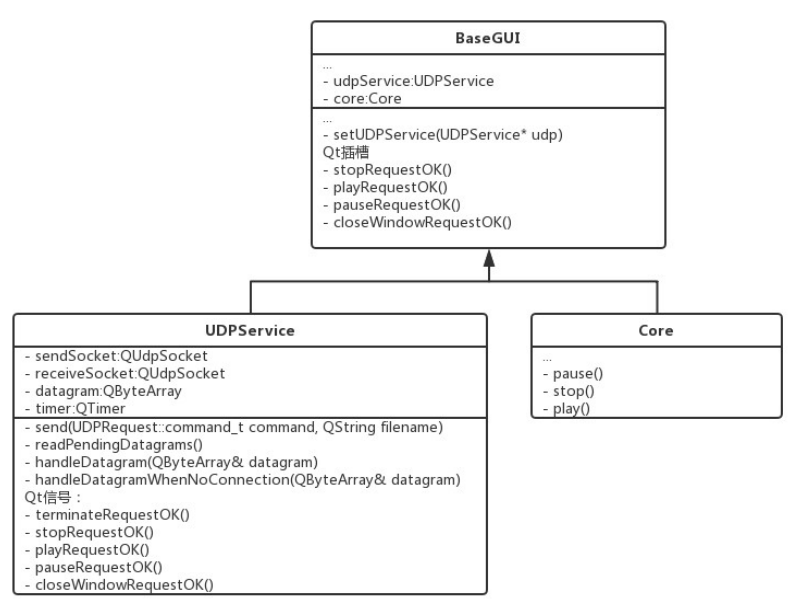

Figure 3. UML class diagram of the $8 \mathrm{~K}$ ultra $\mathrm{HD}$ video and DMS audio broadcast system integration. 
BaseGUI is the common base class of all theme graphical interface. BaseGUI holds the Core and UDPService. BaseGUI is an intermediary of network broadcast and local communication. The relation of these three kinds of classes is shown as Figure 3. BaseGUI initialization connects BaseGUI and UDPService signals and slots by calling void void BaseGui::setUDPService(UDPService* udp). The UDPService's playRequestOK() signal is connected to the BaseGUI's playRequestOK() slot, which causes the Core to start playing, to disable the play button, and to enable the pause button. The UDPService's pauseRequestOK( $)$ signal is connected to the Core's pauseRequestOK() slot, which suspends BaseGUI playback while enabling the play button. The UDPService stopRequestOK() signal is connected to the BaseGUI stopRequestOK () slot, which stops the Core from playing. The terminateRequestOK() signal for UDPService is connected to the BaseGUI closeWindowRequestOK() slot, which notifies the application graphical interface to exit and end the process.

\section{Conclusion}

The $8 \mathrm{~K}$ ultra-high-definition television broadcasting system was developed by Beijing HeXinChenGuang Information Technology Co., Ltd. It can broadcast ultrahigh-definition uncompressed video signals with a bandwidth of $8 \mathrm{~GB} / \mathrm{s}$ and a resolution of $7680 \times 4320$. The Dynamic Matrix Sound (DMS) is a new multi-channel pickup system based on the principle of sound field optimization and the Huygens principle. DMS is developed by the Key Laboratory of Media Audio Education of the Communication University of China. This paper proposes a communication method that integrates the above two systems. It mainly describes an application layer protocol based on User Datagram Protocol (UDP). It focuses on the message distribution and message transmission mechanism of the protocol.
The operating conditions can achieve synchronous playback of $8 \mathrm{~K}$ ultra-high-definition video and DMS audio. The integration application of $8 \mathrm{~K}$ ultra HD video and DMS audio broadcast system is realized.

\section{Acknowledgments}

This work was supported by the Fundamental Research Funds for the Central Universities (No.3132018XNG1844).

\section{References}

1. Xie Xingfu. Principle of Stereo Sound. Beijing: Science Press, 2258-2260 (1981).

2. HAMASAKI K, NISHIGUCHI T, OKUMURA R. A 22. 2 multichannel sound system for Ultra - High Definition TV (UHDTV) [J]. SMPTE Motion Imaging Journal, 2008, 117, (3): 40 -49.

3. Strategy analytics report highlights falling prices, improved upscal-ing capabilities, and greater consumer awareness as key drivers of UHD TV uptake

[EB/OL]. http://www.strategyan-alytics.com/default.aspx?mod $=$ pressreleaseviewer\&a $0=5513,2015$

4. Editorial Department. Japan $8 \mathrm{~K}$ ultra hd television technology research -- Based on 8K Super High Vision (8K SHV) research (1) [J]. Film and television production, 2015, (9): 31.

5. The world's first article 10.5 generation of line production, aimed at $8 \mathrm{~K}$ BOE panel market [EB/OL] http://www.sohu.com/a/211610441 115565, 2017.

6. HAMASAKI K, HIYAMA I K. Development of a 22. 2 multi- channel sound system[J]. Broadcast Technology, 2006, (25): 9-13. 